University of Massachusetts Amherst

ScholarWorks@UMass Amherst

Mathematics and Statistics Department Faculty

Publication Series

2021

\title{
Immune classification of clear cell renal cell carcinoma
}

\author{
Sumeyye Su \\ University of Massachusetts Amherst \\ Shaya Akbarinejad \\ University of Massachusetts Amherst \\ Leili Shahriyari \\ University of Massachusetts Amherst
}

Follow this and additional works at: https://scholarworks.umass.edu/math_faculty_pubs

\section{Recommended Citation}

Su, Sumeyye; Akbarinejad, Shaya; and Shahriyari, Leili, "Immune classification of clear cell renal cell carcinoma" (2021). Scientific Reports. 1308.

https://doi.org/10.1038/s41598-021-83767-z

This Article is brought to you for free and open access by the Mathematics and Statistics at ScholarWorks@UMass Amherst. It has been accepted for inclusion in Mathematics and Statistics Department Faculty Publication Series by an authorized administrator of ScholarWorks@UMass Amherst. For more information, please contact scholarworks@library.umass.edu. 


\title{
OPEN Immune classification of clear cell renal cell carcinoma
}

\author{
Sumeyye Su, Shaya Akbarinejad \& Leili Shahriyari ${ }^{\bowtie}$
}

Since the outcome of treatments, particularly immunotherapeutic interventions, depends on the tumor immune micro-environment (TIM), several experimental and computational tools such as flow cytometry, immunohistochemistry, and digital cytometry have been developed and utilized to classify TIM variations. In this project, we identify immune pattern of clear cell renal cell carcinomas (cCRCC) by estimating the percentage of each immune cell type in 526 renal tumors using the new powerful technique of digital cytometry. The results, which are in agreement with the results of a large-scale mass cytometry analysis, show that the most frequent immune cell types in ccRCC tumors are CD8+ T-cells, macrophages, and CD4+T-cells. Saliently, unsupervised clustering of ccRCC primary tumors based on their relative number of immune cells indicates the existence of four distinct groups of ccRCC tumors. Tumors in the first group consist of approximately the same numbers of macrophages and CD8+ T-cells and and a slightly smaller number of CD4+ T cells than CD8+ T cells, while tumors in the second group have a significantly high number of macrophages compared to any other immune cell type ( $P$-value $<0.01)$. The third group of ccRCC tumors have a significantly higher number of CD8+T-cells than any other immune cell type ( $P$-value $<0.01$ ), while tumors in the group 4 have approximately the same numbers of macrophages and CD4+ T-cells and a significantly smaller number of CD8+ T-cells than CD4+ T-cells ( $P$-value $<0.01$ ). Moreover, there is a high positive correlation between the expression levels of IFNG and PDCD1 and the percentage of CD8+ T-cells, and higher stage and grade of tumors have a substantially higher percentage of CD8+ T-cells. Furthermore, the primary tumors of patients, who are tumor free at the last time of follow up, have a significantly higher percentage of mast cells ( $P$-value $<0.01$ ) compared to the patients with tumors for all groups of tumors except group 3.

Clear cell renal cell carcinoma (ccRCC) is the most frequently diagnosed malignant tumor type in the adult kidneys consisting of approximately $85 \%$ of kidney cancer cases ${ }^{1}$, and surgical resection is the common therapy type for ccRCC. However, it is not effective for patients with advance or metastatic ccRCC ${ }^{2}$. Several immunotherapeutic approaches have been recently used for treating patients with $\mathrm{ccRCC}^{3,4}$, which is considered a morphologically and genetically immunogenic tumor ${ }^{5}$. However, many patients do not respond to these treatments and develop adaptive or intrinsic resistance. We can increase the response rate to these treatments by identifying types of tumors that would respond to them.

Several studies show that cancer cells and tumor infiltrating immune cells (TIICs), which have important roles in both regulation of cancer progression and promotion of tumor development ${ }^{6,7}$, play an important role in the determination of malignant tumor types ${ }^{8,9}$. Tumor-infiltrating lymphocytes (TILs), which include T-cells and B cells, are an important category of TICCs. CD4+ helper T-cells and cytotoxic CD8+ T-cells play a significant role in preventing tumor by targeting antigenic tumor cells ${ }^{10}$, and CD8+ T-cells are linked with better clinical outcomes and reaction to immunotherapy in many cancers ${ }^{11,12}$. Furthermore, it has been recently observed that tumor associated B cells, which have significant roles in the immune system by producing antibodies and presenting antigens, could be predictors of survival and response to immune checkpoint blockade therapy ${ }^{13}$. Additionally, a relationship between TIICs gene signatures and lower survival rates has been observed in ccRCC patients, and tumor-associated macrophages (TAM) and $22 \mathrm{~T}$ cell phenotypes are found to be correlated with clinical outcomes ${ }^{14,15}$. These observations emphasize on importance of analyzing the cellular heterogeneity of tumors, including immune cell variations, to identify target tumors for each specific treatment and design new effective cancer treatments ${ }^{16}$.

There are some experimental approaches such as single cell analysis tools, including immunohistochemistry and flow cytometry to observe tumor immune infiltrates, however these methods are expensive and time consuming, and they are limited to analyzing a few immune cell types simultaneously ${ }^{17}$. For this reason, several 


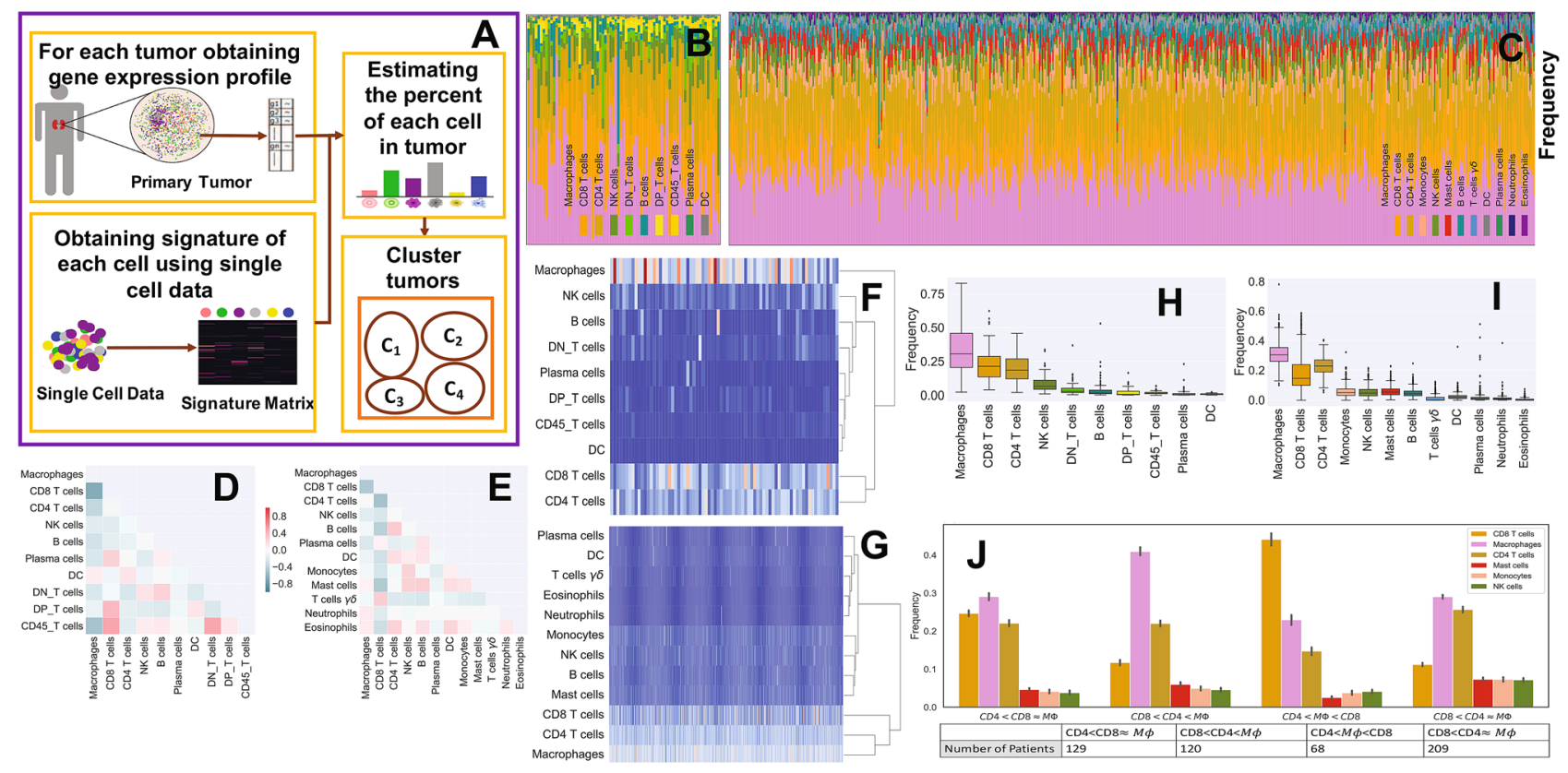

Figure 1. Immune pattern of ccRCC. Sub-figure (A) (created using PowerPoint v16.44) represents the algorithm of digital cytometry and clustering applied on TCGA data. Sub-figures (B) and (C), respectively show the estimated percentage of each immune cell by mass cytometry analysis of 73 ccRCC patients done by Chevrier et al. ${ }^{14}$ (B) and digital cytometry on 526 TCGA ccRCC tumors (C). Sub-figures (D) and (E) indicate the correlation map of estimated immune cell frequencies in 73 ccRCC tumors (D) and TCGA ccRCC tumors (E), respectively. Sub-figures (F) and (G) show the cluster heat map of immune cell frequencies in 73 ccRCC tumors (F) and TCGA ccRCC tumors $(\mathbf{G})$. Sub-figures (H) and (I) respectively show a box plot format of the immune cell percentages in 73 ccRCC tumors (H) and TCGA ccRCC tumors (I). Sub-figure (J) shows 4 distinct immune patterns of ccRCC tumors obtained by K-mean clustering of cell frequencies of TCGA ccRCC tumors. Sub-figures (B)-(I) have been created using TumorDecon ${ }^{19}$ (https://pypi.org/project/TumorDecon/).

computational methods have been recently developed to provide us with much less expensive and fast alternative ways to estimate the relative amount of each cell type from gene expression profiles of bulk tumors. In this study, we applied a powerful "digital cytometry" method called CIBERSORTx ${ }^{18}$ to determine immune patterns of tumors (Fig. 1A) and investigate the association of these patterns with clinical features.

\section{Results}

To estimate the percentage of each cell type in ccRCC tumors, we apply the "digital cytometry" method of CIBERSORTx, which has a good performance ${ }^{19}$, on TCGA gene expression profiles of ccRCC primary tumors. We compare the results of our "digital cytometry" analysis with the results of an experimental study of a large-scale mass cytometry-based immune cells analysis of $73 \mathrm{ccRCC}$ patients ${ }^{14}$. Immune cells, which have been characterized in this experimental study done by Chevrier et al. ${ }^{14}$ are macrophages, CD8+ T-cells, CD4+ T-cells, NK cells, B cells, plasma cells, dendritic cells (DC), CD45+ T-cells, double positive T-cells (DP_T-cells), double negative T-cells (DN_T-cells). To be able to compare our results, which includes 22 immune cell types given in LM22 signature matrix of CIBERSORTx, we combine cells that belong to the same family. For instance, since CD4+ naive T-cell, CD4+ memory resting T-cells, CD4+ memory activated T-cells, follicular helper T-cells, and regulatory T-cells are sub-types of CD4+ T-cells, we sum their numbers to estimate the total number of CD4+ T-cells. We do similar calculation for B cells, NK cells, DC cells, macrophages, and mast cells.

The most frequent immune cells in cCRCC tumors are macrophages, CD4+ T-cells, and CD8+ T-cells. It has been found in the experimental studies that T cells (CD4+ T-cells and CD8+ T-cells) are the main immune cell population in the ccRCC tumors ${ }^{14,20}$. Results of experimental study done by Chevrier et al. ${ }^{14}$ show that macrophages are the most frequent immune cells in most ccRCC tumors with a mean of $31 \%$ followed by $\mathrm{CD} 8+\mathrm{T}$-cells and CD4+ T-cells, respectively $(\mathrm{H})$, which are in agreement with the results of CIBERSORTx applied on TCGA data set (Fig. 1C,I).

There is a negative correlation between the number of macrophages and CD8+ T-cells. The results of mass cytometry analysis indicate a negative correlation between CD8+ T-cells and macrophages with Pearson correlation coefficients of -0.67 . Importantly, the digital cytometry applied on TCGA data set confirms this negative correlation between the number of CD8+ T-cells and macrophages in ccRCC with a correlation coefficient of -0.46 (Fig. 1D,E). 
Variations of ccRCC tumors are mainly in the percentage of macrophages, CD8+ T-cells, and CD4+ T-cells compared to the other immune cell types. Figure 1 shows high variations among the percentage of CD8+ and CD4+ T-cells and macrophages across ccRCC tumors, while there is a slight variation in the percentage of other immune cell types. Unsupervised hierarchical clustering of cell frequencies show that CD8+ T-cells and CD4+ T-cells are clustered together in the experimental results, and then they group with macrophages and other cells (Fig. 1F). The result of digital cytometry on TCGA data shows a kind of similar trend: CD4+ T-cells first clustered with macrophages, then they clustered with CD8+ T-cells and other cells (Fig. 1G).

There are four immune patterns of ccRCCs. K-mean clustering of ccRCC tumors based on their immune cells' frequencies shows that there are four different immune classes: Cluster $1(C D 4<C D 8 \approx M \Phi)$, in which the numbers of macrophages and CD8+ T-cells are approximately the same, and the number of CD4+ T-cells is slightly less than the number of CD8+ T-cells; Cluster 2 called $(C D 8<C D 4<M \Phi)$, in which the number of macrophages is significantly higher than the number of CD4+ and CD8+ T-cells (P-value $<0.01$ ); Cluster $3(C D 4<M \Phi<C D 8)$, in which the number of $C D 8+$ T-cells is significantly higher than the number of macrophages and CD4+ T-cells (P-value $<0.01)$; and Cluster 4 called $(C D 8<C D 4 \approx M \Phi)$ in which the numbers of macrophages and CD4+ T-cells are approximately the same, and the number of CD8+ T-cells is significantly less than CD4+ T-cells (P-value $<0.01$ ) (Fig. 1J).

Cluster $(C D 8<C D 4 \approx M \Phi)$ has the highest percentage of grade $1-2$ and stage T1-T2 tumors. Comparing clinical features of clusters show that Cluster $(C D 8<C D 4 \approx M \Phi)$ includes the highest percentage of grade 1 and grade 2 tumors and the lowest percentage of grade 4 tumors with the chi-squared test's P-value $<0.05$, where the grade of tumor is defined as a numeric value to express the degree of abnormality of cancer cells ${ }^{21}$, and there is a similar trend for the stage of tumors (Fig. 2A,B). Importantly, clusters $(C D 8<C D 4 \approx M \Phi)$ and $(C D 8<C D 4<M \Phi)$ have the highest proportion of patients who were tumor free and smallest percentage of the diseased patients at the last time of follow up among all other clusters (Fig. 2C,D). Furthermore, this cluster has the highest frequency of mast cells, monocytes and B cells compared the other clusters (Fig. 1J). These results might imply that non-aggressive tumors include an approximately equal number of each immune cell type.

Cluster ( $C D 4<M \Phi<C D 8)$ has the highest percentage of grade 4 and stage T4 tumors compared to the other clusters. The result of chi-squared test shows that the percentages of grade 3-4 and stage T3-T4 tumors are significantly higher in Cluster $(C D 4<M \Phi<C D 8)$ compared to the other clusters (Fig. 2A,B, P-value $<0.05$ ). Furthermore, this cluster includes the highest number of deceased patients (chisquared test's P-value $<0.05$ ) and patients who had a tumor at the last time of follow up compared to the other clusters (Fig. 2C,D). Note, patients are categorized as 'tumor free' if they did not have any tumor, and as 'with tumor' if they had any tumor at the last time of follow up ${ }^{21}$. There is a noticeable difference among overall survival months of female and male patients in this cluster, female patients in the cluster $(C D 4<M \Phi<C D 8)$ have the highest overall survival months compared to the other clusters (Fig. $2 \mathrm{H}$ ). These results indicate that male patients' ccRCC tumors consisting of a significantly higher number of CD8+ T-cells than any other immune cell types might be aggressive.

There is no significant differences in overall survival months or age at diagnosis between clusters. Figure 2 indicates no significant differences in the overall survival of patients between any of these clusters; this figure also reveals some other interesting observations. For example, patients in Cluster $C D 4<C D 8 \approx M \Phi$ with and without tumors at the last time of follow up have a similar overall survival months while in all other clusters patients with tumor have a substantially lower survival months than patients without tumors at the last time of follow up (Fig. 2G). Moreover, patients with tumor in this cluster have a remarkably higher age at diagnosis compared to the patients with no tumors in this cluster (Fig. 2J). Furthermore, female patients in this cluster have a noticeably higher age at diagnosis but the same survival as male patients in this cluster (Fig. $2 \mathrm{H}, \mathrm{K}$ ). Additionally, female patients in Cluster $C D 4<M \Phi<C D 8$ have a substantially higher overall survival months than male patients in this cluster, while females have a slightly higher age at diagnosis than males in this cluster. Importantly, there is no significant differences in the age at diagnosis and survival months of patients in each cluster based on the location of their primary tumors, left and right kidneys (Fig. 2I,L).

Higher grade and stage of ccRCC tumors have higher percentage of CD8+ T-cells and lower percentages of mast cells and monocytes. A study of $87 \mathrm{ccRCC}$ patients indicates that the percentage of tumor infiltrating CD8+ T-cells co-expressing PD-1 and Tim-3 is correlated with an aggressive phenotype and a larger tumor size at diagnosis ${ }^{22}$. In another study, it has been found that the grade of ccRCC tumors is an increasing function of CD8+ T cells ${ }^{20}$. Figure 3 also indicates that the grade 3-4 and stage T3-T4 ccRCC tumors have a significantly higher percentage of CD8+ T-cells compared to the stage T1-T2 and grade 1-2 tumors (P-value $<0.01$ ), which is consistent with the observations of Fig. 2.

Figure 3 also indicates that the percentages of mast cells and monocytes in ccRCC tumors significantly decrease when the grade and stage of tumors increase (P-value $<0.01)$. Note, Clusters $(C D 8<C D 4<M \Phi)$ and $(C D 8<C D 4 \approx M \Phi)$ that have higher frequency of mast cells and monocytes and lower frequency of CD8+ T-cells have the least percentage of grade three and four tumors (Figs. 1J and 2). Some studies have reported a correlation between a high density of CD8+ T-cells in RCC patients and shorter overall survival ${ }^{23}$ and worse 

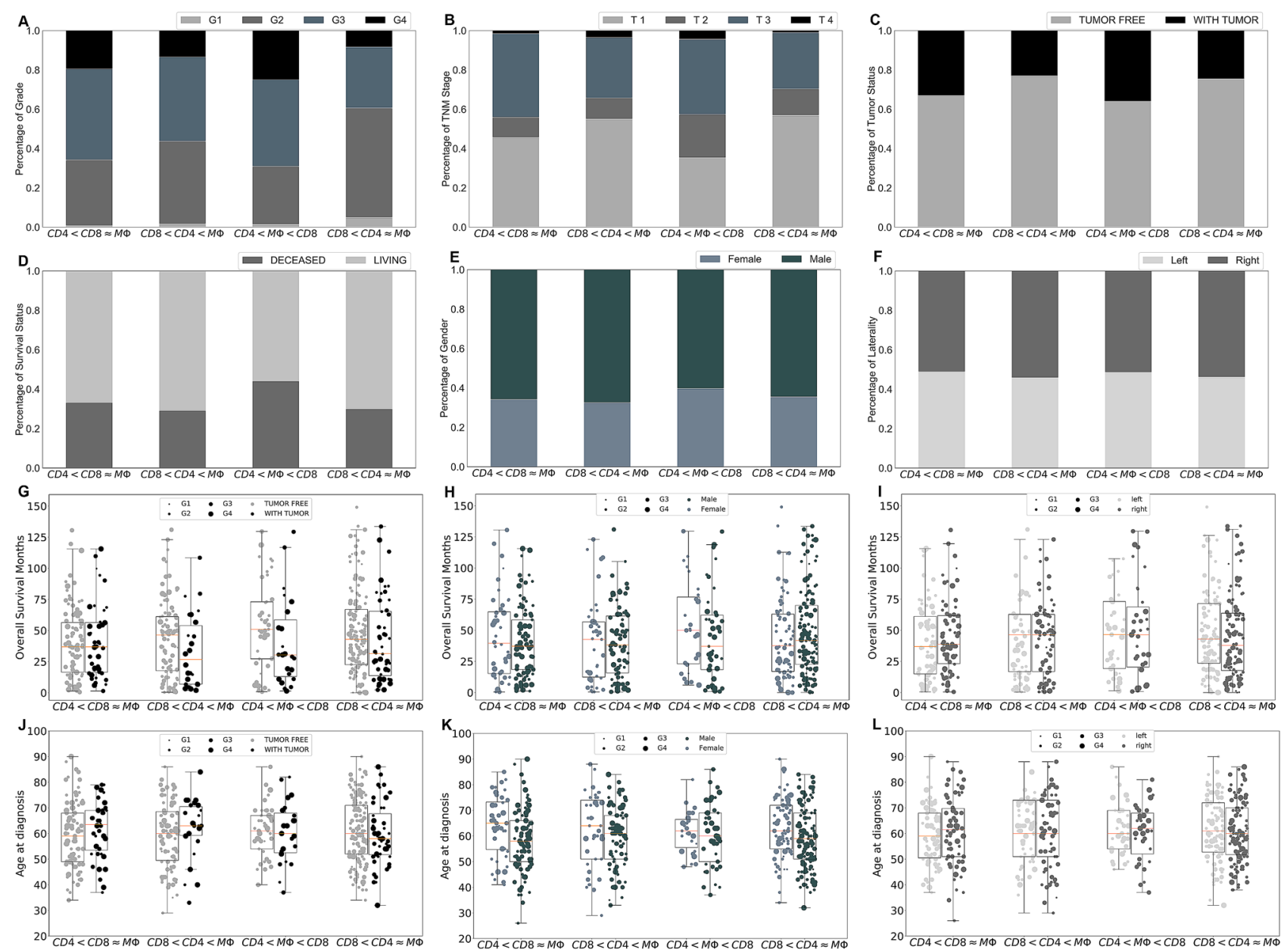

Figure 2. Clinical features of each ccRCC tumor cluster. Sub-figures (A)-(F) show the percentage of patients with grade 1-4 (A), stage T1-T4 (B), with tumors or without tumors (C), alive or dead at the last time of follow up (D), female or male (E), and primary tumors in left or right kidney (F) for each cluster of ccRCC tumors. Sub-figures $(\mathbf{G})-(\mathbf{I})$ and $(\mathbf{J})-(\mathbf{L})$ respectively show the overall survival months and age of diagnosis of the patients in each cluster as a function of tumor status $(\mathbf{G}, \mathbf{J})$, gender $(\mathbf{H}, \mathbf{K})$, and the location of the primary tumor $(\mathbf{I}, \mathbf{L})$; the size of markers indicates the grade of tumors.

treatment response ${ }^{24}$. Similarly, we observe that patients in Cluster $(C D 4<M \Phi<C D 8)$, which has the highest amount of CD8+ T cells, have the worst survival outcome among all clusters (Fig. 2).

Tumor free patients have a significantly higher percentages of mast cells in their primary tumors. NK cells are known for their roles in immune surveillance and destruction of tumor cells $s^{25,26}$. Moreover, flow cytometric and immunohistochemistry analyses show that a high number of NK cells is associated with improved survival ${ }^{23}$ and negatively correlated with the grade of tumor ${ }^{20}$. Also, Fig. 4A shows that primary tumors of patients who are tumor free at the last time of follow up has a significantly higher level of NK cells compared to the patients with tumor (P-value $<0.01$ ). However, a closer look in clusters reveal that the significant difference $(\mathrm{P}$-value $<0.01)$ in percentage of NK cells between tumor free and with tumor patients corresponds to the patients in Cluster $(C D 4<C D 8 \approx M \Phi)$ (Fig. $4 \mathrm{~B})$.

In a recent study, $259 \mathrm{ccRCC}$ patients have been clustered into two groups based on their immunohistochemistry profiles, and it has been observed that patients in the cluster with a high mast cells infiltration have a better response to treatments and a higher survival ${ }^{24}$. In our results, the percentage of mast cells is higher in primary tumors of tumor free patients versus with tumor patients at the last time of follow up in all cluster. Importantly, Cluster $(C D 8<C D 4 \approx M \Phi)$ has the highest percentage of mast cells and NK cells compared to the other clusters (Figs. 1J and 4). Note, this cluster has the highest percentage of grade and stage 1 and 2 tumors. Additionally, ccRCC tumors in Cluster $(C D 4<M \Phi<C D 8)$, which has the highest percentage of grade and stage 4 tumors, have the lowest amount of mast cells.

Genes expression levels of PDCD1 and INFG are significantly positively correlated with the percentage of CD8+ T-cells in ccRCC tumors. Programmed cell death protein 1 (PD-1) is a type of protein that found on T-cells and it prevents T-cells from killing cancer cells when it binds to PD-1 ligand (PD- 

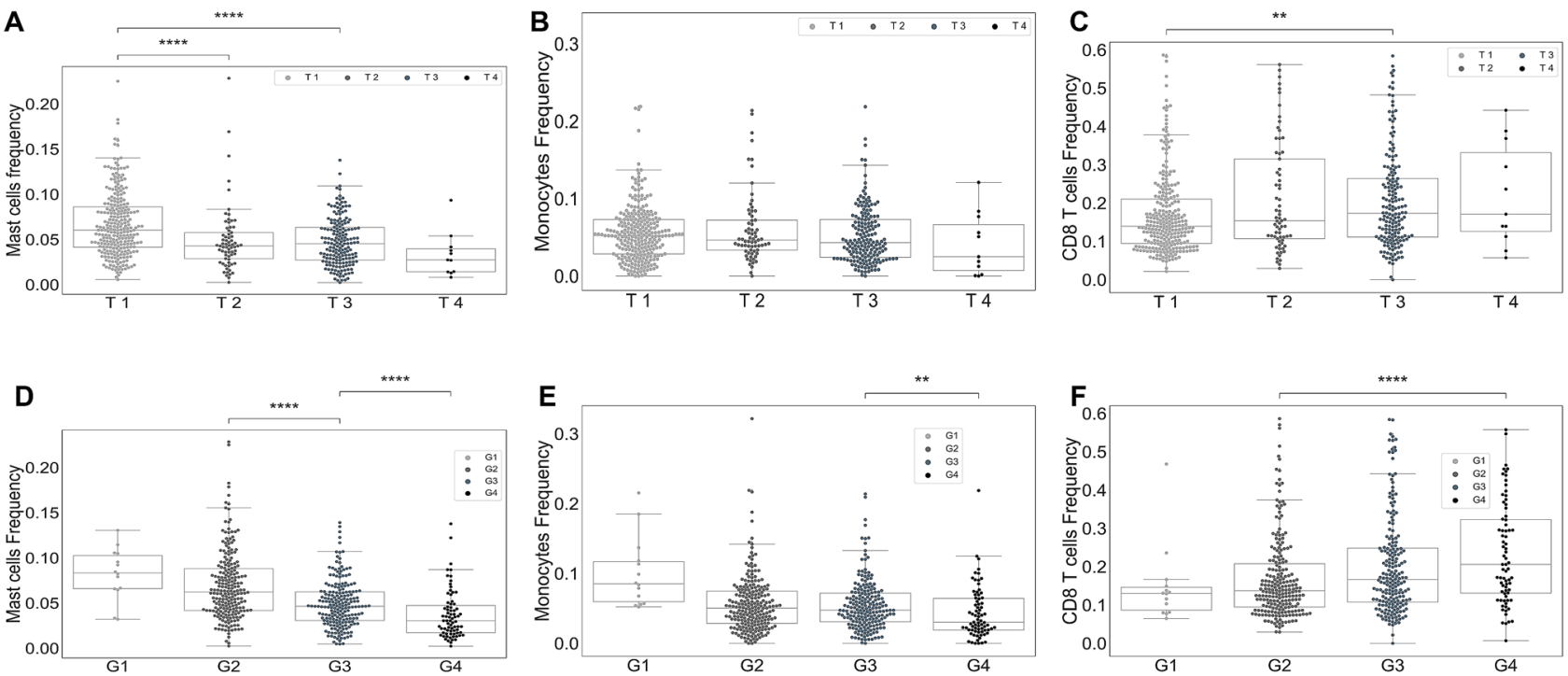

Figure 3. Percentage of mast cells, monocytes and CD8+ T-cells in ccRCC tumors as a function of grade and TNM staging. Sub-figures (A)-(C) show the percentages of mast cells (A), monocytes (B), and CD8+ T-cells (C) in primary tumors as a function of stage of tumors. Sub-figures $(D)-(F)$ represent the percentage of mast cells (D), monocytes (E), and CD8+ T-cells (F) in primary tumors as functions of the grade of tumors.
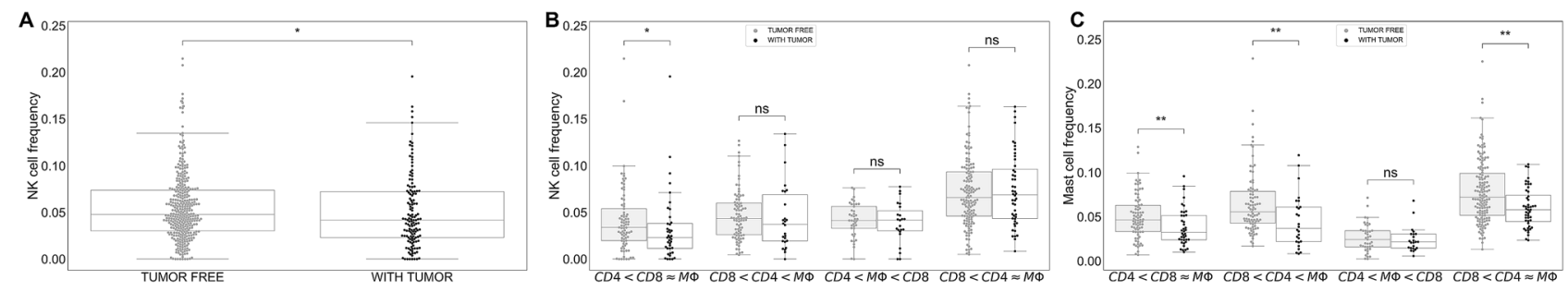

Figure 4. Frequency of NK cells and mast cells in ccRCC. Sub-figure (A) shows that patients who were tumor free at the last time of follow up have higher percentage of NK cells than patients with tumor at the last time. Sub-figures $(\mathbf{B})$ and $(\mathbf{C})$ respectively indicate the percentage of NK cells and mast cells in primary tumors in each cluster.

L1) and PD-2 ligand (PD-L2) on cancer cells. PDCD1 gene, which encodes PD-1 protein, and CD8+ T-cells are highly positively correlated, with correlation coefficient of 0.85 . Also, expression level of PDCD1 is the highest in the cluster $(C D 4<M \Phi<C D 8)$ and the lowest in the cluster $(C D 8<C D 4 \approx M \Phi)$ as a result of positive correlation with CD8+ T-cells (Fig. 5C,E).

Interferon $\gamma(I N F \gamma)$, encoded by INFG gene, is a cytokine that is essential for innate and adaptive immunity. It works as an activator of macrophages and stimulator of $\mathrm{NK}$ cells and neutrophils ${ }^{27}$, and it is mostly produced by T-cells and NK cells as a reaction of a variety of inflammatory or immune stimuli ${ }^{28}$. Saliently, expression level of INFG is significantly positively correlated with the percentage of CD8+ T-cells and the expression level of PDCD1 in ccRCC tumors, with correlation coefficients of 0.79 and 0.87 , respectively. In addition, cluster $(C D 4<M \Phi<C D 8)$ has the highest INFG expression level and cluster $(C D 8<C D 4 \approx M \Phi)$ has the lowest expression level of INFG as expected (Fig. 5).

In contrast, there is a slightly positive correlation between the expression levels of CD274 and PDCD1LG2 genes, that encodes PD-L1 and PD-L2 respectively, with the expression levels of PDCD1 and INFG, and the percentage of CD8+ T-cells in ccRCC tumors (Fig. 5E). In addition, cluster (CD8<CD4 $\approx M \Phi)$ has the lowest levels of CD274 and PDCD1LG2 compared to the other clusters (Fig. 5B,D).

There is a significant association between RGS5 expression level and the percentages of NK cells, monocytes, and mast cells. RGS5 is a member of the regulators of G protein signaling (RGS) family, and they are known as signal transaction molecules that are associated with the arrangement of heterotrimetric G proteins by acting as GTPase activators. Moreover, RGS5 is a hypoxia-inducible factor-1 dependent involved in the induction of endothelial apoptosis. In our previous study on TCGA data, we found that a high expression level of RGS5 in ccRCC primary tumors is associated with better survival months, and when the grade of ccRCC tumor increases, the RGS5 expression level significantly decreases ${ }^{29}$. Interestingly, cluster $(C D 8<C D 4 \approx M \Phi)$ has the highest RGS5 expression level compared to the other clusters, and tumor free patients have a higher level of RGS5 expression than patients with tumor (Fig. 6A). Saliently, ccRCC tumors 
A
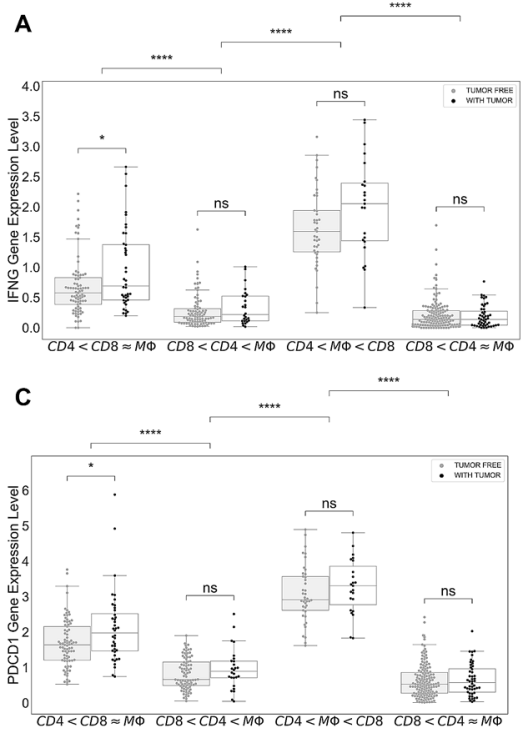

B

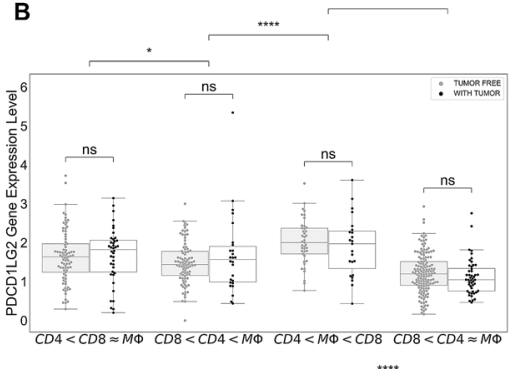

D

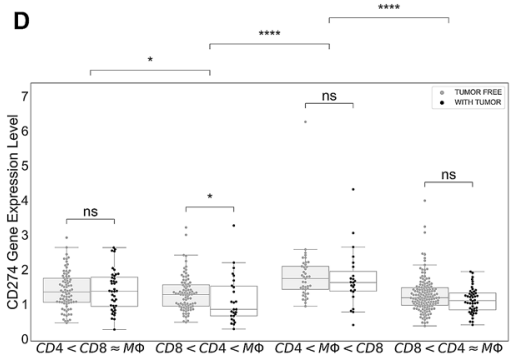

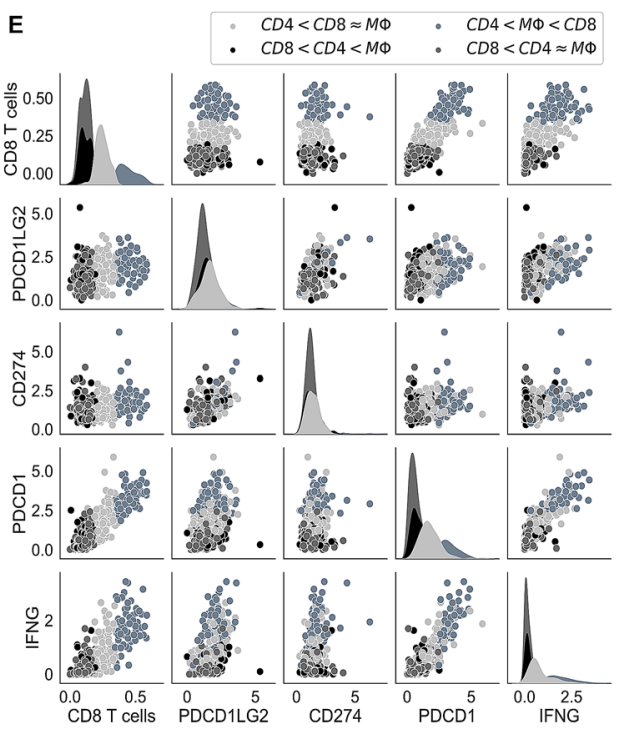

Figure 5. Expression levels of genes encoding PD-1, PD-L1, PD-L2, and IFN $\gamma$. Sub-figures (A)-(D) indicate the expression levels of INFG, PDCD1LG2, PDCD1 and CD274 in each cluster as a function of tumor status, respectively. Sub-figure (E) represents the correlations and distributions of INFG, PDCD1LG2, PDCD1, CD274 expression levels and CD8+ T-cells; color coded based on the clusters.
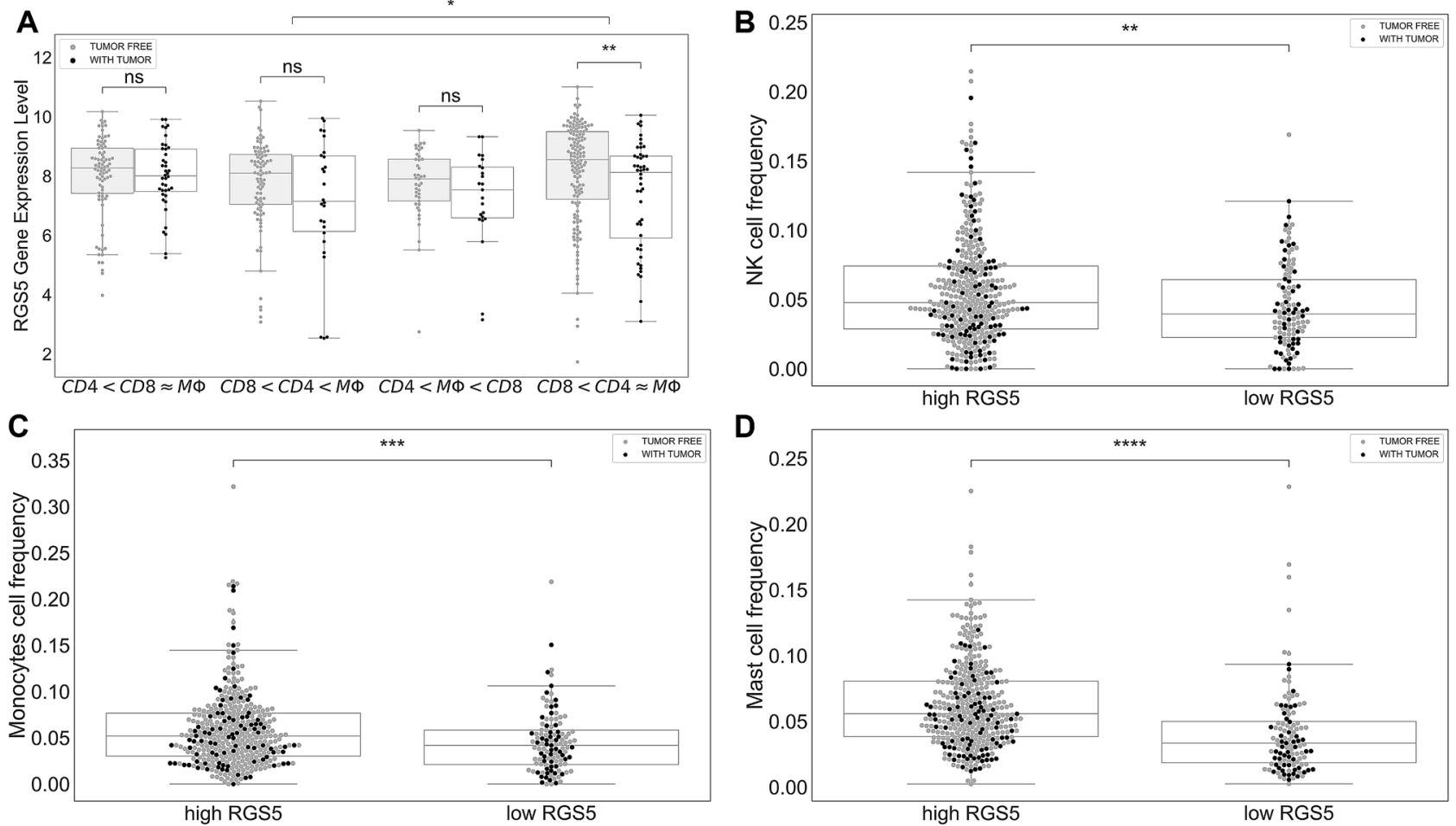

Figure 6. RGS5 expression level in ccRCC tumors. Sub-figure (A) shows the expression level of RGS5 in ccRCC tumors in each cluster as a function of tumor status. Sub-figures (B), (C) and (D) indicate the relation between the level of RGS5 and the percentages of NK cells, monocytes, and mast cells in ccRCC tumors, respectively.

with a high expression level of RGS5 have a significantly high percentages of NK cells, mast cells, and monocytes $($ P-value $<0.01$ ) (Fig. 6B-D).

\section{Discussion}

Immune checkpoints are essential parts of immune system, and they are crucial to prevent autoimmune diseases. However, some tumors benefit from these checkpoints, because these checkpoints can prevent the immune system from killing cancer cells. One such immune checkpoint is programmed cell death 1 (PD-1) protein, which 
binds to its ligand PD-L1 and inhibits immune cell activities, including T cell activities. One strategy for cancer immunotherapy is to block these checkpoints to promote anti-cancer T-cell activities ${ }^{30-33}$. Immunotherapy such as targeting PD-1 pathway has improved overall survival months of several patients with metastatic cancers, including melanoma, head and neck cancer, renal cell carcinoma, non-small cell lung cancer (NSCLC), and colon cancer. However, there are many patients who do not respond to these treatments, and some patients who initially respond to the treatments, they might develop resistance or experience severe adverse events ${ }^{34-38}$. For this reason, further biomarkers of tumor cells such as PD-1 and PD-L1 and of tumor infiltrating immune cells such as T-cells and macrophages need to be established to develop new treatment strategies and identify the patients who can be treated by each drug or treatment strategy ${ }^{39}$.

In kidney cancer, common immunotherapy drugs such as nivolumab and avelumab target PD-1, PD-L1, and PD-L2 pathways ${ }^{40}$. Anti PD-1 drugs targets T-cells directly, while anti-PD-L1 drugs target tumor cells directly, and they may also target tumor associated macrophages that express PD-L1. Several studies indicate an increase of INF $\gamma$ production in the PD-1 inhibitors and other immune checkpoint blockade therapies that resulted in destruction of cancer cells $s^{41-43}$, and a relation between cancer immunotherapy improvement and an increase of $I N F \gamma$ expression has been observed ${ }^{28}$. Moreover, a correlation observed between an increase in INF $\gamma$ gene expression and better progression-free survival in NSCLC and urothelial cancer patients treated with a PD-L1 inhibitor $^{44}$.

Note, tumors in cluster $(C D 4<M \Phi<C D 8)$ have a high expression levels of INFG, the gene encoding $I N F \gamma$, and PDCD1, the gene encoding PD-1, compared to the other clusters, and the expression levels of these genes are significantly positively correlated with the percentage of CD8+ T-cells in tumors. Importantly, it has been shown that INF $\gamma$ boosts the CD8+ T-cells expansion ${ }^{45}$. Thus, patients in the cluster $(C D 4<M \Phi<C D 8)$ might respond to the PD-1 inhibitors. In addition, since there is not a strong correlation between PDCD1LG2 and CD274 expression levels and levels of INFG and PDCD1 genes, PD-L1 and PD-L2 inhibitors might not be as effective treatments as the PD- 1 inhibitors for the patients in this cluster. Although Cluster $(C D 8<C D 4 \approx M \Phi)$ includes a high number of patients with lower grade and without tumor in the last follow up time, tumors in this cluster have lower levels of INFG and PDCD1, therefore patients in this cluster may not be a good candidate for anti PD-1 therapies.

Anti-angiogenic agent $(\mathrm{AA})$ is one of the main treatments in the aggressive $\mathrm{ccRCC}^{1}$, because nutrients and oxygen are the main ingredients of the tumor growth which come from blood. Anti-angiogenics, also known angiogenesis inhibitors, are drugs that stop the growth of blood vessels (angiogenesis) that tumors need to grow ${ }^{46}$. A study of in vitro cell lines and in vivo mouse models of ccRCC shows that the recruitment of mast cells is related with increased ccRCC angiogenesis by modulating $P I 3 K \rightarrow A K T \rightarrow G S K 3 \beta \rightarrow A M$ signaling pathway ${ }^{47}$. Since Cluster $(C D 8<C D 4 \approx M \Phi)$ has the highest amount of mast cells compared to the other clusters, angiogenesis inhibitors might be a good treatment option for the patients in this cluster. Moreover, mast cells are suggested as an independent prognostic factor in some studies of ccRCC patients ${ }^{48,49}$. It has been observed that the number of mast cells is negatively correlated with 5 -year survival ${ }^{49}$ and positively correlated with grade, pT stage, and metastasis ${ }^{50}$. Contradicting these observations, a recent study of ccRCC patients shows that an increased mast cells infiltration is linked with better treatments' responses and survival ${ }^{24}$. We have similarly observed that the number of mast cells is inversely correlated with the grade of tumors (Fig. 3A,D), and the primary tumors of patients without tumors at the last time of follow up have higher percentages of mast cells than primary tumors of patients with tumor at the last time of follow up.

Kruger et al. ${ }^{51}$ suggested RGS5 gene as a tumor associated antigenes (TAAs), and they observed over-expressed RGS5 level from a large scale analysis of ccRCC specimens. Another study found that RGS5 is strongly up-regulated in a broad variety of malignant cells and showed that RGS5 peptides might be a good candidate for designing cancer vaccines to target malignant cells and tumor vessels ${ }^{52}$. We found that patients with higher RGS5 levels have significantly higher percentages of NK cells, mast cells, and monocytes in their primary tumors (P-value $<0.01)$. Moreover, patients in Cluster $(C D 8<C D 4 \approx M \Phi)$ have the highest amount of RGS5 expression in their primary tumor. With the help of further investigation, RGS5 gene might be a good target for patients in this cluster. Further clinical and biological studies are required to test and validate all above mentioned suggestions.

\section{Materials and methods}

We estimated the percentage of tumor infiltrating immune cells in ccRCC tumors using CIBERSORTx deconvolution method that is based on the following linear model:

$$
A X=b,
$$

where $b$, which is called mixture data, is the gene expression profile of the bulk tumor, and $X$ is unknown cell proportions in $b$. A, which is called signature matrix, is the gene expression profile of cells.

In the first version of CIBERSORT, a machine learning technique, Nu-Support Vector Regression ( $v$-SVR), is used to solve the problem $(1)^{53}$. Matrix $A$ in Eq. (1) is determined by a hyperplane with capturing the data points inside an $\varepsilon$-tube that is determined by support vectors (genes in signature matrix). SVR penalizes the data points outside the $\varepsilon$-tube, and a small value is used for $v$ that determines the lower bound of support vectors and the upper bound of training errors. Regression coefficients of $\nu$-SVR method are the values of the vector $X$. However, the proportions are non-negative values, and their sum must be one. Therefore, negative coefficients are set to 0 , and they normalize the remaining coefficients to sum to $1^{53}$. Newman et al. ${ }^{18}$ have recently improved their method by adding batch correction modes to remove possible cross-platform variations between signature matrix and mixture data.

To investigate the immune variations in renal cancer, we downloaded TCGA data set ${ }^{54}$ of gene expression profiles of 607 ccRCC primary tumors from UCSC Xena ${ }^{55}$ to use as a mixture data $b$. We used LM22 signature 


\begin{tabular}{|l|l|l|l|l|l|l|l|l|l|l|}
\hline Gender & \multicolumn{4}{|l|}{ Grade of tumor } & \multicolumn{4}{|l|}{ Stage of tumor } \\
\hline Female & Male & G1 & G2 & G3 & G4 & T1 & T2 & T3 & T4 \\
\hline 184 & 342 & 14 & 226 & 204 & 75 & 268 & 69 & 178 & 11 \\
\hline Tumor status & \multicolumn{1}{l|l|l|l|l|l|l|l|l|l|}{} \\
\hline Tumor free & With tumor & Alive & Deceased & Left & \multicolumn{2}{|l|}{ Right } \\
\hline 356 & 137 & 355 & 171 & 247 & 287 & \\
\hline
\end{tabular}

Table 1. Patients' characteristics. Sub-tables indicate the number of patients in each category. Differences in the numbers are due to missing information for some patients.

matrix, which includes normalized expression levels of 547 gene signatures that are filtered against other normal and cancer cell types to differentiate 22 cell types that are naive B cells, memory B cells, Plasma cells, CD8+ T-cells, CD4+ naive T-cells, CD4+ memory resting T-cells, CD4+ memory activated T-cells, follicular helper T-cells, regulatory T-cells (Tregs), $\gamma \delta$ T-cells, resting NK cells, activated NK cells, monocytes, M0 macrophages, M1 macrophages, M2 macrophages, resting dendritic cells, activated dendritic cells, resting mast cells, activated mast cells, eosinophils, neutrophils ${ }^{53}$. We then estimated cell fractions in ccRCC tumors using CIBERSORTx B-mode to remove technical differences between LM22 signature matrix and TCGA RNA-seq data. Note, genes that are used to identify each type of immune cells in LM22 signature matrix can be found in the supplementary file of CIBERSORT paper ${ }^{53}$.

After we estimated cell proportions, we included only cases with CIBERSORTx P-value $<0.05$. We then applied unsupervised K-mean clustering algorithm to cluster tumors based on their percentage of immune cells. The K-mean algorithm separates samples in k-group of equal variance by minimizing the inertia (distance between samples in the clusters and center of the clusters). To determine the optimal number of clusters (k-value), we used elbow method to find the best value for $k^{56}$.

We also collected clinical information of patients from $\mathrm{cBioPorta}^{57}$ and dropped some patients due to missing clinical information and continued our analysis with 526 patients. Patients' characteristic are given in Table 1.

For statistical analyses, we used the non-parametric Mann-Whitney-Wilcoxon (MWW) test between groups of continuous variables, because values in the comparison groups are not normally distributed and there are different numbers of patients in the comparison groups. MWW tests whether the values in one of two comparison groups is significantly larger than the other ${ }^{58}$. We also used chi-squared test to determine whether there is a statistically significant difference between the frequencies of the categorical variables. Stars in the figures show the significance levels where, ns: $0.05<P \leq 1,{ }^{*}: 0.01<P \leq 0.05,{ }^{* *}: 0.001<P \leq 0.01,{ }^{* *}: 0.0001<P \leq 0.001$, $* * * *: P \leq 0.0001$.

Ethics. No ethical approval was required for this study.

\section{Data availability}

The TCGA data ${ }^{54}$ underlying this article are available at https://www.cbioportal.org/datasets ${ }^{57}$ and https://xenab rowser.net/datapages $/{ }^{55}$.

Received: 26 May 2020; Accepted: 5 February 2021

Published online: 22 February 2021

\section{References}

1. Choueiri, T. K. \& Motzer, R. J. Systemic therapy for metastatic renal-cell carcinoma. N. Engl. J. Med. 376, 354-366. https://doi. org/10.1056/NEJMra1601333 (2017).

2. Climent, M. A., Muñoz-Langa, J., Basterretxea-Badiola, L. \& Santander-Lobera, C. Systematic review and survival meta-analysis of real world evidence on first-line pazopanib for metastatic renal cell carcinoma. Crit. Rev. Oncol. Hematol. 121, 45-50. https:// doi.org/10.1016/j.critrevonc.2017.11.009 (2018).

3. Escudier, B. Emerging immunotherapies for renal cell carcinoma. Ann. Oncol. 23, viii35-viii40. https://doi.org/10.1093/annonc/ mds261 (2012).

4. Considine, B. \& Hurwitz, M. E. Current status and future directions of immunotherapy in renal cell carcinoma. Curr. Oncol. Rep. 21, 34. https://doi.org/10.1007/s11912-019-0779-1 (2019).

5. Linehan, W. M. \& Zbar, B. Focus on kidney cancer. Cancer Cell 6, 223-228. https://doi.org/10.1016/j.ccr.2004.09.006 (2004).

6. Grivennikov, S. I., Greten, F. R. \& Karin, M. Immunity, inflammation, and cancer. Cell 140, 883-899. https://doi.org/10.1016/j. cell.2010.01.025 (2010).

7. Kitamura, T., Qian, B.-Z. \& Pollard, J. W. Immune cell promotion of metastasis. Nat. Rev. Immunol. 15, 73-86. https://doi. org/10.1038/nri3789 (2015).

8. Candido, J. \& Hagemann, T. Cancer-related inflammation. J. Clin. Immunol. 33, 79-84. https://doi.org/10.1007/s10875-012-9847-0 (2013).

9. Swann, J. B. \& Smyth, M. J. Immune surveillance of tumors. J. Clin. Investig. 117, 1137-1146. https://doi.org/10.1172/JCI31405 (2007).

10. Vesely, M. D., Kershaw, M. H., Schreiber, R. D. \& Smyth, M. J. Natural innate and adaptive immunity to cancer. Annu. Rev. Immunol. 29, 235-271. https://doi.org/10.1146/annurev-immunol-031210-101324 (2011).

11. Yao, J. et al. Checkpoint molecule PD-1-assisted CD8+ T lymphocyte count in tumor microenvironment predicts overall survival of patients with metastatic renal cell carcinoma treated with tyrosine kinase inhibitors. Cancer Manag. Res. 10, 3419-3431. https ://doi.org/10.2147/CMAR.S172039 (2018). 
12. Pagès, F. et al. In situ cytotoxic and memory T cells predict outcome in patients with early-stage colorectal cancer. J. Clin. Oncol. 27, 5944-5951. https://doi.org/10.1200/JCO.2008.19.6147 (2009).

13. Griss, J. et al. B cells sustain inflammation and predict response to immune checkpoint blockade in human melanoma. Nat. Commun.https://doi.org/10.1038/s41467-019-12160-2 (2019).

14. Chevrier, S. et al. An immune atlas of clear cell renal cell carcinoma. Cell 169, 736-749.e18. https://doi.org/10.1016/j.cell.2017.04.016 (2017).

15. Ricketts, C. J. et al. The cancer genome atlas comprehensive molecular characterization of renal cell carcinoma. Cell Rep. 23, 313326.e5. https://doi.org/10.1016/j.celrep.2018.03.075 (2018).

16. Becht, E., Giraldo, N. A., Dieu-Nosjean, M. C., Sautès-Fridman, C. \& Fridman, W. H. Cancer immune contexture and immunotherapy. Curr. Opin. Immunol. 39, 7-13. https://doi.org/10.1056/NEJMra16013334 (2016).

17. Heath, J. R., Ribas, A. \& Mischel, P. S. Single-cell analysis tools for drug discovery and development. Nat. Rev. Drug Discov. 15, 204-216. https://doi.org/10.1038/nrd.2015.16 (2016).

18. Newman, A. M. et al. Determining cell type abundance and expression from bulk tissues with digital cytometry. Nat. Biotechnol. 37, 773-782. https://doi.org/10.1056/NEJMra16013336 (2019).

19. Le, T., Aronow, R. A., Kirshtein, A. \& Shahriyari, L. A review of digital cytometry methods: Estimating the relative abundance of cell types in a bulk of cells. Brief. Bioinform.https://doi.org/10.1093/bib/bbaa219 (2020).

20. Geissler, K. et al. Immune signature of tumor infiltrating immune cells in renal cancer. OncoImmunology 4, e985082. https://doi. org/10.1056/NEJMra16013337 (2015).

21. National Cancer Institute. GDC Documentation. https://docs.gdc.cancer.gov/Data_Dictionary/viewer/\#?view=table-definition -view\&id=diagnosis.

22. Granier, C. et al. Tim-3 expression on tumor-infiltrating PD-1 + CD8 + T cells correlates with poor clinical outcome in renal cell carcinoma. Cancer Res. 77, 1075-1082. https://doi.org/10.1158/0008-5472.CAN-16-0274 (2017).

23. Remark, R. et al. Characteristics and Clinical Impacts of the Immune Environments in Colorectal and Renal Cell Carcinoma lung metastases: Influence of tumor origin. Clin. Cancer Res. 19, 4079-4091. https://doi.org/10.1158/1078-0432.CCR-12-3847 (2013).

24. Xiong, Y. et al. Identification and validation of dichotomous immune subtypes based on intratumoral immune cells infiltration in clear cell renal cell carcinoma patients. J. ImmunoTher. Cancer 8, e000447. https://doi.org/10.1136/jitc-2019-000447 (2020).

25. Vivier, E., Tomasello, E., Baratin, M., Walzer, T. \& Ugolini, S. Functions of natural killer cells. Nat. Immunol. 9, 503-510. https:// doi.org/10.1016/j.critrevonc.2017.11.0092 (2008).

26. Levy, E. M., Roberti, M. P. \& Mordoh, J. Natural killer cells in human cancer: From biological functions to clinical applications. J. Biomed. Biotechnol. 1-11, 2011. https://doi.org/10.1155/2011/676198 (2011).

27. Tau, G. \& Rothman, P. Biologic functions of the IFN- $\gamma$ receptors. Allergy Eur. J. Allergy Clin. Immunol. 54, 1233-1251. https://doi. org/10.1034/j.1398-9995.1999.00099.x (1999).

28. Ni, L. \& Lu, J. Interferon gamma in cancer immunotherapy. Cancer Med. 7, 4509-4516. https://doi.org/10.1002/cam4.1700 (2018).

29. Su, S. \& Shahriyari, L. RGS5 plays a significant role in renal cell carcinoma. R. Soc. Open Sci. 7, 191422. https://doi.org/10.1098/ rsos.191422 (2020).

30. Chen, D. S. \& Mellman, I. Oncology meets immunology: The cancer-immunity cycle. Immunity 39, 1-10. https://doi.org/10.1016/j. critrevonc.2017.11.0096 (2013).

31. Motzer, R. J. et al. Nivolumab versus everolimus in advanced renal-cell carcinoma. N. Engl. J. Med. 373, 1803-1813. https://doi. org/10.1056/NEJMoa1510665 (2015).

32. Dine, J., Gordon, R., Shames, Y., Kasler, M. \& Barton-Burke, M. Immune checkpoint inhibitors: An innovation in immunotherapy for the treatment and management of patients with cancer. Asia-Pac. J. Oncol. Nurs. 4, 127. https://doi.org/10.4103/apjon.apjon _4_17 (2017).

33. Motzer, R. J. et al. Nivolumab plus ipilimumab versus sunitinib in advanced renal-cell carcinoma. N. Engl. J. Med. 378, 1277-1290. https://doi.org/10.1056/NEJMoa1712126 (2018).

34. Gettinger, S. N. et al. Overall survival and long-term safety of nivolumab (anti-programmed death 1 antibody, BMS-936558, ONO-4538) in patients with previously treated advanced non-small-cell lung cancer. J. Clin. Oncol. 33, 2004-2012. https://doi. org/10.1007/s11912-019-0779-10 (2015).

35. Koyama, S. et al. Adaptive resistance to therapeutic PD-1 blockade is associated with upregulation of alternative immune checkpoints. Nat. Commun. 7, 1-9. https://doi.org/10.1038/ncomms10501 (2016).

36. Hofmann, L. et al. Cutaneous, gastrointestinal, hepatic, endocrine, and renal side-effects of anti-PD-1 therapy. Eur. J. Cancer 60, 190-209. https://doi.org/10.1007/s11912-019-0779-12 (2016).

37. Wu, X. et al. Application of PD-1 blockade in cancer immunotherapy. Comput. Struct. Biotechnol. J. 17, 661-674. https://doi. org/10.1016/j.csbj.2019.03.006 (2019).

38. Sharpe, A. H. \& Pauken, K. E. The diverse functions of the PD1 inhibitory pathway. Nat. Rev. Immunol. 18, 153-167. https://doi. org/10.1007/s11912-019-0779-14 (2018).

39. Maleki Vareki, S., Garrigós, C. \& Duran, I. Biomarkers of response to PD-1/PD-L1 inhibition. Crit. Rev. Oncol. Hematol. 116, 116-124. https://doi.org/10.1016/j.critrevonc.2017.06.001 (2017).

40. Aggen, D. H., Drake, C. G. \& Rini, B. I. Targeting PD-1 or PD-L1 in metastatic kidney cancer: Combination therapy in the first-line setting. Clin. Cancer Res.https://doi.org/10.1158/1078-0432.CCR-19-3323 (2020).

41. Chen, H. et al. Anti-CTLA-4 therapy results in higher CD4 + ICOS hi T cell frequency and IFN- $\gamma$ levels in both nonmalignant and malignant prostate tissues. Proc. Nat. Acad. Sci. 106, 2729-2734. https://doi.org/10.1007/s11912-019-0779-15 (2009).

42. Dulos, J. et al. PD-1 Blockade Augments Th1 and Th17 and Suppresses Th2 Responses in Peripheral Blood From patients with prostate and advanced melanoma cancer. J. Immunother. 35, 169-178. https://doi.org/10.1097/CJI.0b013e318247a4e7 (2012).

43. Peng, W. et al. PD-1 blockade enhances T-cell migration to tumors by elevating IFN-inducible chemokines. Cancer Res. 72 , 5209-5218. https://doi.org/10.1158/0008-5472.CAN-12-1187 (2012).

44. Higgs, B. W. et al. Interferon gamma messenger RNA signature in tumor biopsies predicts outcomes in patients with non-small cell lung carcinoma or urothelial cancer treated with durvalumab. Clin. Cancer Res. 24, 3857-3866. https://doi.org/10.1158/10780432.CCR-17-3451 (2018).

45. Sercan, Ö., Hämmerling, G. J., Arnold, B. \& Schüler, T. Cutting Edge: Innate Immune Cells Contribute to the IFN- $\gamma$-Dependent Regulation of Antigen-specific CD8 + T cell homeostasis. J. Immunol. 176, 735-739. https://doi.org/10.4049/jimmunol.176.2.735 (2006).

46. Al-Husein, B., Abdalla, M., Trepte, M., DeRemer, D. L. \& Somanath, P. R. Antiangiogenic therapy for cancer: An update. Pharmacother. J. Hum. Pharmacol. Drug Ther. 32, 1095-1111. https://doi.org/10.1002/phar.1147 (2012).

47. Chen, Y. et al. Infiltrating mast cells promote renal cell carcinoma angiogenesis by modulating PI3K $\rightarrow A K T \rightarrow G S K 3 \beta \rightarrow A M$ signaling. Oncogene 36, 2879-2888. https://doi.org/10.1016/j.ccr.2004.09.0060 (2017).

48. Fu, H. et al. Tumor infiltrating mast cells (TIMs) confers a marked survival advantage in nonmetastatic clear-cell renal cell carcinoma. Ann. Surg. Oncol. 24, 1435-1442. https://doi.org/10.1245/s10434-016-5702-5 (2017).

49. Cherdantseva, T. M. et al. Mast cells in renal cancer: Clinical morphological correlations and prognosis. Bull. Exp. Biol. Med. 163, 801-804. https://doi.org/10.1007/s10517-017-3907-7 (2017).

50. Nakanishi, H. et al. Pathological significance and prognostic roles of densities of CD57+ cells, CD68+ cells, and mast cells, and their ratios in clear cell renal cell carcinoma. Hum. Pathol. 79, 102-108. https://doi.org/10.1016/j.ccr.2004.09.0063 (2018). 
51. Krüger, T. et al. Lessons to be learned from primary renal cell carcinomas: Novel tumor antigens and HLA ligands for immunotherapy. Cancer Immunol. Immunother. 54, 826-836. https://doi.org/10.1016/j.ccr.2004.09.0064 (2005).

52. Boss, C. N. et al. Identification and characterization of T-cell epitopes deduced from RGS5, a novel broadly expressed tumor antigen. Clin. Cancer Res. 13, 3347-55. https://doi.org/10.1158/1078-0432.CCR-06-2156 (2007).

53. Newman, A. M. et al. Robust enumeration of cell subsets from tissue expression profiles. Nat. Methods 12, 453. https://doi. org/10.1016/j.ccr.2004.09.0066 (2015).

54. Network, C. G. A. R. et al. Comprehensive molecular characterization of clear cell renal cell carcinoma. Nature 499, 43 (2013).

55. Goldman, M. J. et al. Visualizing and interpreting cancer genomics data via the Xena platform. Nat. Biotechnol. 38, 675-678. https ://doi.org/10.1016/j.ccr.2004.09.0067 (2020).

56. Arthur, D. \& Vassilvitskii, S. K-means++: The advantages of careful seeding. In Proceedings of the 18th Annual ACM-SIAM Symposium on Discrete Algorithms (2007).

57. Cerami, E. et al. The CBIO cancer genomics portal: An open platform for exploring multidimensional cancer genomics data. Cancer Discov. 2, 401-404. https://doi.org/10.1158/2159-8290.CD-12-0095 (2012).

58. Mann, H. B. \& Whitney, D. R. On a test of whether one of two random variables is stochastically larger than the other. Ann. Math. Stat. 18, 50-60. https://doi.org/10.1214/aoms/1177730491 (1947).

\section{Acknowledgements}

Research reported in this publication was supported by the National Cancer Institute of the National Institutes of Health under Award Number R21CA242933.

\section{Author contributions}

S.S. performed the analysis, analysed the results, and wrote the manuscript. S.A. prepared the data. L.S. designed and supervised the project, and participated in writing the manuscript. All authors reviewed the manuscript.

\section{Competing interests}

The authors declare no competing interests.

\section{Additional information}

Correspondence and requests for materials should be addressed to L.S.

Reprints and permissions information is available at www.nature.com/reprints.

Publisher's note Springer Nature remains neutral with regard to jurisdictional claims in published maps and institutional affiliations.

Open Access This article is licensed under a Creative Commons Attribution 4.0 International License, which permits use, sharing, adaptation, distribution and reproduction in any medium or format, as long as you give appropriate credit to the original author(s) and the source, provide a link to the Creative Commons licence, and indicate if changes were made. The images or other third party material in this article are included in the article's Creative Commons licence, unless indicated otherwise in a credit line to the material. If material is not included in the article's Creative Commons licence and your intended use is not permitted by statutory regulation or exceeds the permitted use, you will need to obtain permission directly from the copyright holder. To view a copy of this licence, visit http://creativecommons.org/licenses/by/4.0/.

(C) The Author(s) 2021 